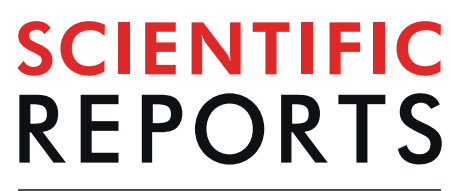

\title{
Southeastern Asia fall armyworms are closely related to populations in Africa and India, consistent with common origin and recent migration
}

\author{
Rodney N. Nagoshi ${ }^{*}$, Ni Ni Htain ${ }^{2}$, Duncan Boughton ${ }^{3}$, Lei Zhang $\mathbb{1}^{4}$, Yutao Xiao ${ }^{4}$, \\ Benjamin Y. Nagoshi ${ }^{5}$ \& David Mota-Sanchez ${ }^{6}$
}

The discovery of fall armyworm, a native of the Western Hemisphere, in western Africa in 2016 was rapidly followed by detections throughout sub-Saharan Africa, India, and most recently southeastern Asia. This moth pest has a broad host range that threatens such important crops as corn, rice, millet, and sorghum, creating concern for its potential impact on agriculture in the Eastern Hemisphere. Although genetic data suggest populations sampled in Africa and India originate from a recent common source, it is not known whether this is the case for populations in southeastern Asia, nor whether the subgroup with a preference for rice and millet is present in the region. This study found through comparisons of genetic markers that the fall armyworm from Myanmar and southern China are closely related to those from Africa and India, suggesting a common origin for these geographically distant populations. The results are consistent with a single recent introduction into the Eastern Hemisphere followed by rapid dispersion. The molecular similarities include discrepancies between the genetic markers that brings into question whether the subpopulation most likely to be a threat to rice and millet is present in significant numbers in Asia.

The fall armyworm (Spodoptera frugiperda; J. E. Smith)(Lepidoptera: Noctuidae) is a significant economic pest of corn and other crops in the Western Hemisphere and is noted for its broad host range (over 80 host plant species reported) and long-distance migration capability ${ }^{1}$. These characteristics have become a global concern with the introduction of fall armyworm into the Eastern Hemisphere and its spread from western Africa to southeastern Asia over a remarkably short period of time. Large infestations of fall armyworm in Africa were first reported in southwestern Nigeria in $2016^{2}$. Within the next two years infestations were observed in most sub-Saharan nations ranging from Kenya to the east and South Africa to the south ${ }^{3-7}$. In 2018 fall armyworm populations were found in multiple locations in India ${ }^{8-11}$, and now most recently in southeastern Asia ${ }^{12-14}$.

Conjectures about fall armyworm movements in the Eastern Hemisphere depend on whether the timing of first detections in various regions accurately reflect the first arrival of the pest. If so, then fall armyworm in two years traversed a minimum of 7,000 km from Nigeria to India and then another $4,000 \mathrm{~km}$ in the next year to southeastern Asia. If this occurred through natural migration it would seemingly require movements over large bodies of water, desert, and other habitats where the primary host plants would be expected to be scarce. Fall armyworm does migrate thousands of kilometers annually in North America, but this occurs with very favorable wind patterns and a plentiful supply of corn acreage along the migration routes ${ }^{15}$. It therefore seems likely that

\footnotetext{
${ }^{1}$ Center for Medical, Agricultural and Veterinary Entomology, United States Department of Agriculture-Agricultural Research Service, Gainesville, Florida, United States of America. ${ }^{2}$ Biological Control Section, Plant Protection Division, Yangon, Myanmar. ${ }^{3}$ Department of Agricultural, Food and Resource Economics, Michigan State University, East Lansing, Michigan, United States of America. ${ }^{4}$ Agricultural Genomics Institute at Shenzhen, Chinese Academy of Agricultural Sciences, Shenzhen, 518120, China. ${ }^{5}$ University of South Florida, Tampa, Florida, United States of America. ${ }^{6}$ Department of Entomology, Michigan State University, East Lansing, Michigan, United States of America. *email: rodney.nagoshi@usda.gov
} 
if the dispersion of fall armyworm in the Eastern Hemisphere began in 2016 from a western Africa entry point, human transport and commerce played a significant role to facilitate the movements. If correct, this scenario would indicate a remarkable susceptibility of the Eastern Hemisphere to invasions by exotic migratory moth pests. Understanding how such population movements occurred will become critical to mitigating future such occurrences.

An alternative possibility is that fall armyworm has long been endemic in the Eastern Hemisphere but was undetected until 2016. In this case, the enhanced monitoring occurring as a consequence of its discovery is giving the illusion of rapid migration. However, the limited amount of genetic variation found in the Eastern Hemisphere populations so far tested and the genetic homogeneity between the fall armyworm in India and Africa are not compatible with this proposal ${ }^{16}$. Instead, they suggest a recent and common origin for these geographically distant populations. Whether this is also the case with the southeastern Asia fall armyworm populations has not been tested to our knowledge.

A second area of concern and uncertainty is whether both strains of fall armyworm are present in the Eastern Hemisphere and in particular Asia. The broad host range exhibited by the species is in part due to the presence of two subpopulations that differ in their host plant preferences. Originally labelled as "host strains" the groups were named after the crop upon which they were first identified, with the "rice-strain" in subsequent studies preferentially found in pasture grass and millet, while the "corn-strain" predominates in corn and sorghum ${ }^{17-19}$. Although initially identified on rice, the specificity of the rice-strain to this host appears to be more variable and therefore uncertain $^{20}$. Because the host specificities of the two strains are still being determined we will from this point refer to the corn-strain and rice-strain by the more generic terms $\mathrm{C}$-strain and R-strain, respectively.

A complicating factor particularly in field studies is that the strains are for all practical purposes morphologically indistinguishable, with molecular markers the most reliable diagnostic tool. Specifically, the biased host plant distribution of the C-strain and R-strain populations show a consistent but not absolute correspondence with genetic polymorphisms in the mitochondrial cytochrome oxidase subunit I (COI) and nuclear triosephosphate isomerase (Tpi) genes that themselves are generally, but not always, in agreement ${ }^{17,21,22}$. Typically about $20 \%$ of larvae collected directly from corn plants and adult males from pheromone traps placed in corn fields display R-strain diagnostic molecular markers ${ }^{23-25}$.

An unusual feature of the collections so far analyzed from Africa and India is that as defined by the COI marker the R-strain is the predominant form in most locations even though all collections tested to date came from C-strain preferred hosts ${ }^{5,6,16}$. However, when the same collections were tested for the Tpi marker, $>95 \%$ of the specimens were identified as C-strain. The correspondence of the Tpi identification with host plant and its disagreement with COI suggests that COI may not be an accurate strain marker in Africa and India. If true then the presence of the R-strain is in question in the Eastern Hemisphere, an important consideration for risk assessments given the importance of R-strain preferred crops in many Asian countries.

There are two objectives to this study. The first is to assess the similarity of the fall armyworms found in southeast Asia with those from Africa and India to estimate the likelihood that they are part of the same invasion event. The second is to determine whether the disagreement between the COI and Tpi strain markers observed in Africa and India is also a characteristic of southeast Asian fall armyworm. We discuss the implications of the results to our understanding of fall armyworm movements in the Eastern Hemisphere and the risk posed by this pest on R-strain preferred crops (such as rice and millet) in Asia.

\section{Methods}

Specimen collections and dna preparation. Larval collections were made in 2018 from eight provinces in Myanmar and subdivided into three groups approximating lower Myanmar (Ayeyarwaddy, Mon, and Kayin), upper Myanmar (Nay Pyi Taw, Kayah, Magwe, and Mandalay), and hilly regions (Kachin). Identification of fall armyworm specimens was performed using morphological criteria ${ }^{14}$. In a separate survey of China, collections were made by pheromone trapping of adult males in Yunnan province and larval collections in Guangxi, Guangdong and Hunan provinces during March to May in 2019 (Fig. 1a). Collected specimens were stored dry or in ethanol. There are numerous lepidopteran pests of corn reported in southeastern Asia that potentially complicates the identification of fall armyworm ${ }^{26}$. Therefore, fall armyworm identity for all Asian specimens was confirmed by $\mathrm{COI}$ sequence analysis. Collections and data from previous studies include larval collections from Florida $^{21}$, Argentina ${ }^{17}$, India ${ }^{16}$, and Africa ${ }^{5}$.

Larvae from Myanmar were processed using a 5-ml Dounce homogenizer (Thermo Fisher Scientific, Waltham, MA, USA) in $800 \mu \mathrm{l}$ Genomic Lysis buffer (Zymo Research, Orange, CA, USA). The homogenate was incubated at $55^{\circ} \mathrm{C}$ for $15-30 \mathrm{~min}$, then centrifuged at $10,000 \mathrm{rpm}$ for $5 \mathrm{~min}$. DNA was purified using a Zymo-Spin III column (Zymo Research, Orange, CA, USA) and processed according to manufacturer's instructions. Genomic DNA preparations were stored at $-20^{\circ} \mathrm{C}$. Species identity was initially estimated by larval morphology and confirmed by $C O I$ sequence analysis.

Larvae from China were ground with liquid nitrogen and genomic DNAs extracted with Axyprep Multisource Genomic DNA Miniprep Kit (Corning, Corning, NY) according to manufacturer's instructions. Genomic DNA were stored at $-20^{\circ} \mathrm{C}$ before analysis. Species were identified according to morphological characteristics.

PCR amplification and DNA sequencing. Polymerase chain reaction (PCR) amplification occurred in a $30 \mu \mathrm{l}$ reaction mix with a final concentration of $1 \mathrm{X}$ ThermoPol reaction buffer and 0.75 units Taq DNA polymerase (both from New England Biolabs, Beverly, MA), $0.17 \mathrm{mM}$ dNTP, and $0.3 \mu \mathrm{M}$ of each primer. The PCR protocol was $94^{\circ} \mathrm{C}(1 \mathrm{~min})$, followed by 30 cycles of $92^{\circ} \mathrm{C}(30 \mathrm{~s}), 58^{\circ} \mathrm{C}(30 \mathrm{~s}), 72^{\circ} \mathrm{C}(45 \mathrm{~s})$, and a final segment of $72^{\circ} \mathrm{C}$ for $3 \mathrm{~min}$. Primers were synthesized by Integrated DNA Technologies (Coralville, IA). Amplification of the COIB segment was done with primers $c 924 F\left(5^{\prime}\right.$-TTATTGCTGTACCAACAGGT- $\left.3^{\prime}\right)$ and $c 1303 R\left(5^{\prime}\right.$ CAGGATAGTCAGAATATCGACG-3 $3^{\prime}$ ). Samples that gave poor amplification were reanalyzed using nested PCR 


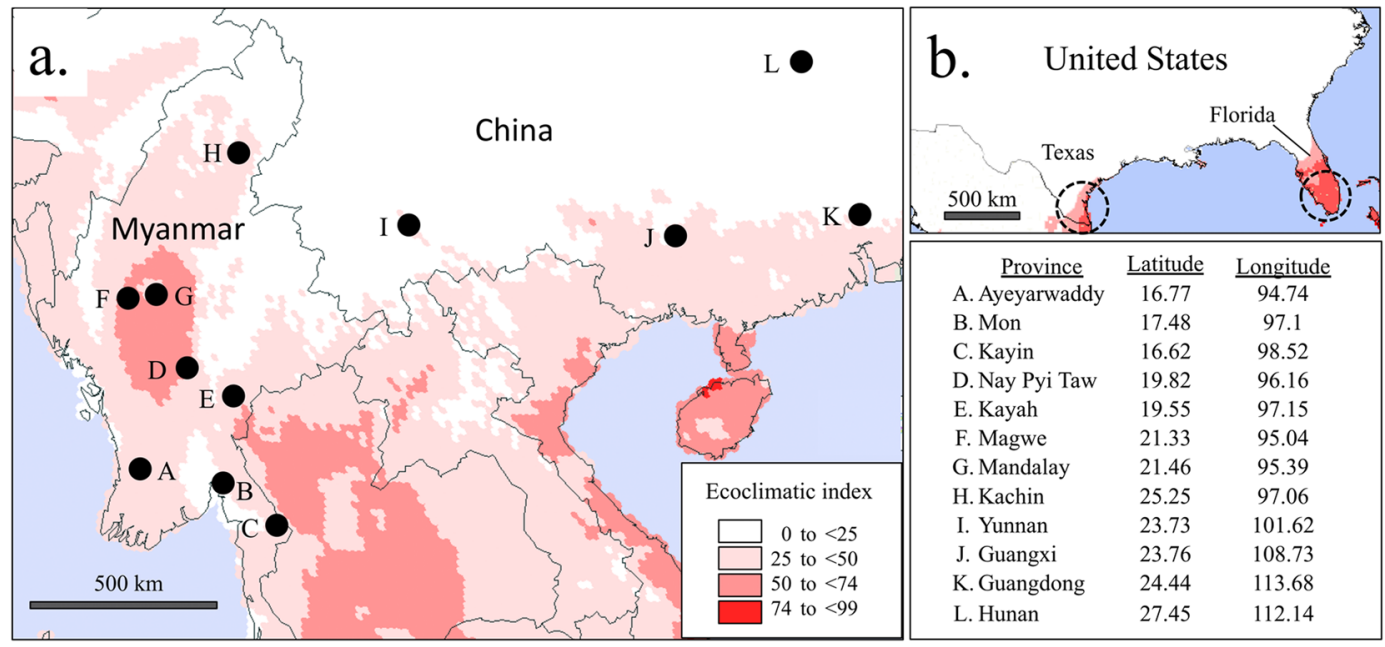

Figure 1. Map and coordinates of collection sites in Myanmar and China combined with CLIMEX modeling of area suitability for fall armyworm. Collection (i) describes pheromone trapping of adult males. All others represent larval collections from corn host plants. (a) locations of sites overlaid on CLIMEX projection of fall armyworm suitability based on calculations of the Ecoclimatic Index (EI), with higher values indicating greater likelihood of persistent fall armyworm populations. (b) CLIMEX projections for the southeastern United States with the same parameters used in Asia. Circles indicate approximate regions where fall armyworm populations are localized during the winter in the United States based on monitoring studies.

in which the first amplification was performed using primers $c 891 F\left(5^{\prime}\right.$-TACACGAGCATATTTTACATC-3') and $c 1472 R\left(5^{\prime}\right.$-GCTGGTGGTAAATTTTGATATC-3') followed by a second PCR using the internal primers $c 924 F$ and $c 1303 R$. Amplification of the Tpi segment was done with primers $t 412 F\left(5^{\prime}-\right.$ CCGGACTGAAGGTTATCGCTTG - $\left.3^{\prime}\right)$ and $11140 R$ ( $5^{\prime}$ - GCGGAAGCATTCGCTGACAACC-3'). Nested PCR was also used, with the first PCR done with primers $t 634 F\left(5^{\prime}\right.$-TTGCCCATGCTCTTGAGTCC- $\left.3^{\prime}\right)$ and $t 1166 R$ ( $5^{\prime}$-TGGATACGGACAGCGTTAGC-3') and the second PCR using the internal primers $t 412 \mathrm{~F}$ and $t 1140 R$.

For gel electrophoresis, $6 \mu \mathrm{l}$ of $6 \mathrm{X}$ gel loading buffer was added to each amplification reaction and the entire sample run on a 1.8\% agarose horizontal gel containing GelGreen (Biotium, Hayward, CA) in 0.5X Tris-borate buffer (TBE, $45 \mathrm{mM}$ Tris base, $45 \mathrm{mM}$ boric acid, $1 \mathrm{mM}$ EDTA pH 8.0). Fragments were visualized on a blue light box and excised from the gel. DNA purification was performed using Zymo-Spin I columns (Zymo Research, Orange, CA) according to manufacturer's instructions. Genewiz (South Plainfield, NJ) performed the DNA sequencing.

DNA alignments and consensus building were performed using MUSCLE (multiple sequence comparison by log-expectation), a public domain multiple alignment software. Phylogenetic trees were constructed using the Tamura-Nei genetic distance model and the UPGMA tree building network ${ }^{27}$. These programs are incorporated into the Geneious Pro 10.1.2 program (Biomatters, New Zealand, http://www.geneious.com) ${ }^{28}$.

Characterization of the $\mathrm{COI}$ and Tpi gene segments. The COI and Tpi strain diagnostic markers are single nucleotide substitutions. Site designations begin with an "m" (mitochondria) or "g" (genomic). This is then followed in order by the gene name, number of base pairs from the predicted translational start site (for COI) or the $5^{\prime}$ start of the exon $(T p i)$, and finally the observed polymorphism using IUPAC convention ( $\mathrm{R}=\mathrm{A}$ or $\mathrm{G} ; \mathrm{Y}=\mathrm{C}$ or $\mathrm{T} ; \mathrm{W}=\mathrm{A}$ or $\mathrm{T} ; \mathrm{K}=\mathrm{G}$ or $\mathrm{T} ; \mathrm{S}=\mathrm{C}$ or $\mathrm{G} ; \mathrm{D}=\mathrm{A}$ or $\mathrm{G}$ or $\mathrm{T}$ ).

The COIB segment was amplified by primers $c 924 F$ and $c 1303 R$. Species identity of the Myanmar specimens was confirmed by sequence comparisons of COIB259, a 259-bp segment common to the GenBank sequences for the following Spodoptera species, S. abula (HQ177287), S. cosmiodes (HQ177295), S. descoinsi (HQ177306), S. dolichos (HQ177313), S. eridania (Stoll in Cramer)(HQ177321), S. exempta (Walker)(HQ177334), S. exigua (Hübner)(HQ177339), S. latisfscia (Walker)(HQ177354), S. littoralis (Boisduval)(HQ177364), S. mauritia (Boisduval)(HQ177382), S. ornithogalli (Guenée)(HQ177392), S. praefica (Grote)(HQ177407), S. litura (F.) (HQ177375). Sites $\mathrm{mCOI1164D}$ and $\mathrm{mCOI} 1287 \mathrm{R}$ are diagnostic for strain identity in Western Hemisphere populations where there is a single rice-strain, $\mathrm{T}_{1164} \mathrm{~A}_{1287}$, and four corn-strain configurations, $\mathrm{A}_{1164} \mathrm{~A}_{1287}(\mathrm{~h} 1)$, $\mathrm{A}_{1164} \mathrm{G}_{1287}$ (h2), $\mathrm{G}_{1164} \mathrm{~A}_{1287}$ (h3), and $\mathrm{G}_{1164} \mathrm{G}_{1287}$ (h4) ${ }^{29}$ (Fig. 2a).

The Tpi Exon-4 segment consists of multiple strain specific polymorphisms with the gTpi183Y site considered diagnostic of strain identity (Fig. 2b). A C 183 identifies the C-strain allele, TpiC, while $\mathrm{T}_{183}$ defines the R-strain, $\mathrm{TpiR}^{21}$. The Tpi gene is located on the $Z$ sex chromosome that is present in one copy in females and two copies in males, with the latter providing opportunities for heterozygosity. Because the genomic DNA was directly sequenced, males heterozygous for Tpi alleles will simultaneously display both alternatives at polymorphic sites, which if different can be identified by overlapping sequencing chromatographs. Heterozygosity at site gTpi183Y gave rise to an overlapping $\mathrm{C}$ and $\mathrm{T}$ signal at gTpi183Y. This was designated $\mathrm{TpiH}$ and defined as representing a TpiC/TpiR heterozygote. 

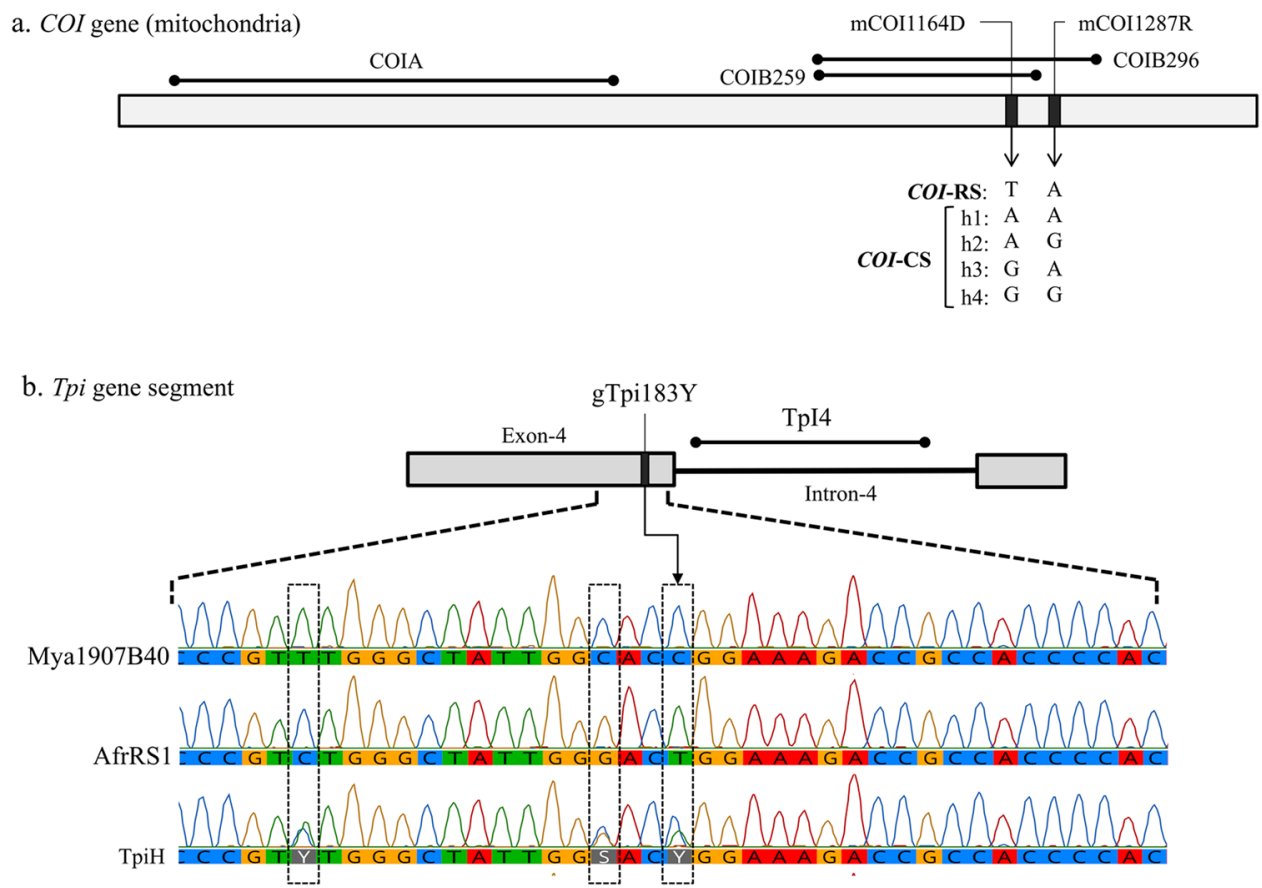

Figure 2. Diagrams of relevant regions in the COI and Tpi genes used for molecular analysis. (a) the COI gene segments with locations of polymorphic sites used for categorizing the COI-CS h1-h4 variants. (b) Map of the Tpi gene segment consisting of the fourth exon of the presumptive open reading frame and adjacent intron. Site gTpi183Y defines the Tpi-based strain identity. Below are chromatographs for the exon segment containing gTpi183Y and two other strain-specific polymorphic sites. Mya1907B40 is a TpiC allele found in Myanmar while AfrRS1 is the TpiR allele identified in Africa. Combining the two produces the overlapping chromatograph pattern found with TpiH.

A portion of the adjacent $T p i$ intron was previously used in phylogenetic comparisons ${ }^{30,31}$. An approximately 172-bp segment of the intron (TpI4) beginning 10-bp from the $5^{\prime}$ splice site was used in the analysis, with the length variable because of indels. This segment was chosen because it displayed the most consistent sequence quality with the given primers. Many samples examined were heterozygous for frameshift mutations within the intron that produced overlapping chromatographs beginning at the polymorphism. These were not further analyzed. Sequences deposited in GenBank include Mya1907c28 (MN551267), Mya1910a85 (MN551268), Mya1907d14 (MN551269), Mya1910B28 (MN551270), AfrCala (MN551271), AfrCa2a (MN551272), AfrCa2b (MN551273), AfrCa1b (MN551274), AfrCa2c (MN551275), and AfrRala (MN551276).

Calculation of haplotype numbers. Specimens have a single mitochondrial COI haplotype and so frequency was calculated as the number of specimens with a given COI haplotype divided by the total number of specimens. The Tpi marker is more complicated because of the potential for heterozygosity. Specimens can be characterized by three Tpi strain categories, TpiC (C-strain), TpiR (R-strain), and TpiH (TpiC/TpiR heterozygote). Frequencies at the specimen level were calculated by the number of each category divided by the total number of specimens. We also calculated the frequency of Tpi chromosomes, which allows inclusion of the TpiH specimens when estimating the number of Tpi alleles. Larvae were not sexed so those identified as TpiC or TpiR could have one (females) or two (males) copies of the Tpi gene. We accounted for this uncertainty by assuming a 1:1 sex ratio and using 1.5 as the mean number of Tpi genes per TpiC or TpiR specimen based on the formula of [2 (Tpi genes in males) +1 (Tpi gene in females)]/2. The TpiH specimens were presumed to carry one copy each of TpiC and TpiR. From these considerations we derived the following formulae, TpiC (chromosomes) $=1.5 \mathrm{X}$ TpiC (specimens) + TpiH and TpiR (chromosomes) $=1.5 \mathrm{X}$ TpiR (specimens) + TpiH. Chromosome frequency was calculated by dividing the number of TpiC or TpiR chromosomes by the total number of chromosomes, as determined by the equation Total chromosomes $=1.5(\mathrm{TpiC}+\mathrm{TpiR}$ specimens $)+2(\mathrm{TpiH}$ specimens $)$.

CLIMEX climate suitability analysis. CLIMEX estimates the potential geographical distribution and relative abundance of a species based on biological parameters and regional climate conditions ${ }^{32}$. The biological parameter values for fall armyworm were previously published (Table 1$)^{33}$. Climate information was imported from Climond (www.climond.org) ${ }^{32,34}$ for selected regions using historical data from 1961-1990 at a resolution of 10 feet.

The Ecoclimatic Index (EI) integrates projected growth potential counterbalanced by estimates of stress, the latter of which is based primarily on unfavorable temperature and moisture conditions. EI is presented on a 0-100 scale, where 100 represent continuous 100\% suitability (as in an incubator). For this study, the Compare Locations (1 species) function in the CLIMEX program was used with the Grid Data simulation file. No climate change scenario or irrigation components were set. An EI map was created from the simulation. 


\begin{tabular}{|c|c|c|}
\hline Parameter & Description & Value \\
\hline \multicolumn{3}{|l|}{$\underline{\text { Moisture }}$} \\
\hline SM0 & Lower soil moisture threshold & 0.1 \\
\hline SM1 & Lower optimal soil moisture & 0.7 \\
\hline SM2 & Upper optimal soil moisture & 0.9 \\
\hline SM3 & Upper soil moisture threshold & 1.5 \\
\hline \multicolumn{3}{|c|}{$\underline{0.5 \text { Temperature }}$} \\
\hline DV0 & Lower temperature threshold & $12^{\circ} \mathrm{C}$ \\
\hline DV1 & Lower optimal temperature & $22^{\circ} \mathrm{C}$ \\
\hline DV2 & Upper optimal temperature & $27^{\circ} \mathrm{C}$ \\
\hline DV3 & Upper temperature threshold & $34^{\circ} \mathrm{C}$ \\
\hline \multicolumn{3}{|l|}{ Cold Stress } \\
\hline TTCS & Cold stress temperature threshold & $8^{\circ} \mathrm{C}$ \\
\hline THCS & Cold stress accumulation rate & -0.001 week $^{-1}$ \\
\hline \multicolumn{3}{|l|}{$\underline{\text { Heat Stress }}$} \\
\hline TTHS & Heat stress temperature threshold & $38^{\circ} \mathrm{C}$ \\
\hline THHS & Heat stress accumulation rate & 0.001 week $^{-1}$ \\
\hline \multicolumn{3}{|l|}{$\underline{\text { Dry Stress }}$} \\
\hline SMDS & Soil moisture dry stress threshold & 0.1 \\
\hline HDS & Dry stress accumulation rate & -0.001 week $^{-1}$ \\
\hline \multicolumn{3}{|l|}{$\underline{\text { Wet Stress }}$} \\
\hline SMWS & Soil moisture wet stress threshold & 1.5 \\
\hline HWS & Wet stress accumulation rate & 0.001 week $^{-1}$ \\
\hline \multicolumn{3}{|c|}{ Minimum degree-day sum needed to complete a generation } \\
\hline PDD & Degree-Days per generation & $559^{\circ} \mathrm{C}$ \\
\hline
\end{tabular}

Table 1. CLIMEX parameter values used for modelling fall armyworm.

\section{Results}

Climate suitability projections. Locations with climatic conditions suitable for fall armyworm populations in the surveyed region were determined by CLIMEX analysis (Fig. 1a). Ecoclimatic index (EI) values were calculated with areas with an EI equal to or greater than 25 indicated in red with darker shading indicating higher values. The higher the EI value, the greater the likelihood of persistent fall armyworm populations, with values greater than 30 considered to be high suitability ${ }^{32}$. All but one collection site was in the vicinity of suitable habitats. To assess the accuracy of the CLIMEX analysis the same parameters were used to analyze the southeastern United States for fall armyworm suitability. Two United States locations were identified, in southern Texas and Florida (Fig. 1b), approximately consistent with estimates of the overwintering range derived from pest monitoring ${ }^{1,35}$. These results indicate that the surveyed locations include areas with a high probability of supporting permanent fall armyworm populations and so are potential sources of migratory populations.

Characterization of Myanmar fall armyworm using COI. The mitochondrial COI and nuclear Tpi genes carry polymorphisms used to characterize fall armyworm populations (Fig. 2). Sequence analysis of the COIB259 segment (COIB259) from COI identified five haplotypes from 106 specimens collected from eight provinces in Myanmar. Phylogenetic comparisons with sequences from 13 Spodoptera species confirmed the fall armyworm identification of the Myanmar specimens and identified two C-strain (COI-CS) and three R-strain (COI-RS) variants (Fig. 3a). Three Myanmar COIB sequences (Mya1907a73, Mya1911b88, and Mya1907b34) are identical to haplotypes found in Africa, while Mya1907b06 and Mya1910c06 differ by only a single base change from the closest Africa variants.

The two most common Myanmar COIB259 haplotypes, Mya1907a73 (AfrRSa1) and Mya1907b34 (AfrCSa1) accounted for more than 96\% (102/106) of the Myanmar collections (Fig. 3b). These are also the most frequent haplotypes in Africa, where the relative proportions observed in pooled collections from eastern Africa (Burundi, Kenya, and Tanzania) were similar to that observed in Myanmar.

Although the Myanmar collections were from corn only a minority of $19 \%$ (22/106) displayed the COI-CS haplotype of the C-strain that is associated with corn preference. This differs from what is typically observed in the Western Hemisphere as indicated by pooled collections from corn hosts in Florida and Argentina (Fig. 3b). A comparison of these collections reveals a regional haplotype bias where AfrCSa1 is the majority COI-CS haplotype in Florida while AfrCSa2 predominates in Argentina. All 22 of the COI-CS specimens so far examined from Myanmar are AfrCSa1. Additional sequence analysis was performed (COIB296) to allow analysis of sites $m C O I 164 \mathrm{D}$ and $\mathrm{mCOI} 1287 \mathrm{R}$ that in combination produce sequence variants with geographical differences in distribution (h1-h4, Fig. 2a) ${ }^{36}$. All COI-CS specimens from Myanmar expressed the h4 combination of $\mathrm{G}_{1164} \mathrm{G}_{1287}$, which is the subgroup most commonly found in Florida and the Caribbean ${ }^{29}$ and a result similar to that observed in India and Africa ${ }^{16,30}$ 
a.
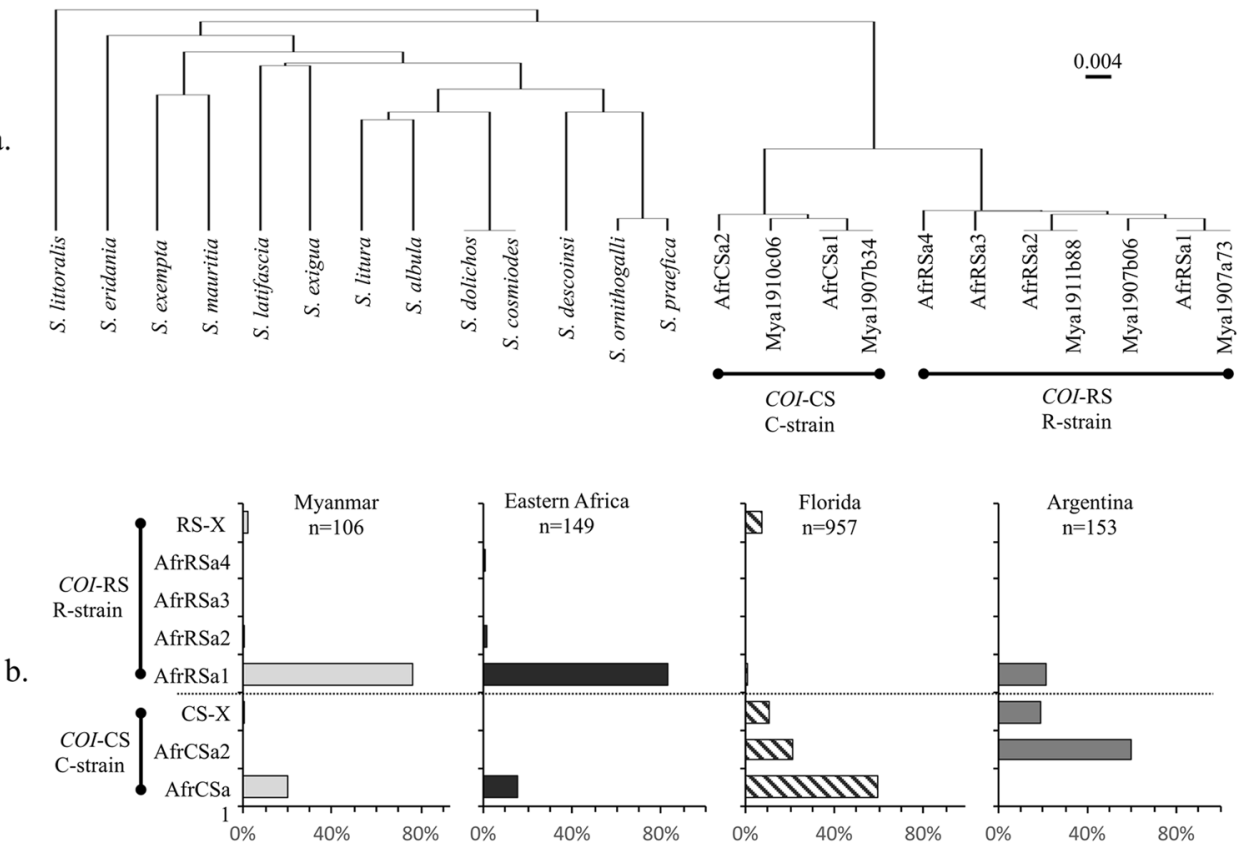

Figure 3. Phylogenetic tree calculated for the COIB259 sequence using the UPGMA method and TamuraNei modeling together with comparisons of haplotype frequencies from different regions. (a) Comparison of COIB259 haplotypes from Myanmar (MyaXXXXXXX) compared to 13 Spodoptera species (from GenBank) and haplotypes observed in Africa (AfrXXXX). (b) COI-CS variants that differ from those observed in Africa.

Characterization of Myanmar fall armyworm using Tpi. The distributions of the COI and Tpi strain markers in the Western Hemisphere are exemplified by larval collections from Florida ${ }^{21}$ where the C-strain COI-CS and TpiC markers generally predominate in specimens from corn and sorghum but are a minority in R-strain hosts such as pasture grasses (Fig. 4). In the Myanmar collections, pooled data from the southern provinces (A-C from Fig. 1a), central provinces (D-G), and Kachin province (I) showed a majority COI-RS and TpiC expression, a pattern also found in eastern Africa and India (Fig. 4).

The TpiR sequence was not directly detected in the Myanmar collections (Fig. 5). This included an additional 92 specimens analyzed for the Tpi markers for a total sample size of 198 . The great majority of specimens were $\mathrm{TpiC}(169)$ and with the remainder TpiH. ${ }^{29}$ However, it is likely that the TpiH class is made up of heterozygotes carrying both TpiR and TpiC. This is indicated by the TpiH DNA sequence chromatographs where the pattern of overlapping signals that can be explained by the presence of both $\mathrm{TpiC}$ and $\mathrm{TpiR}$ haplotypes (Fig. 2b). In this specific case, overlaying the most common TpiC haplotype in Myanmar with the most frequent TpiR haplotype found in Africa (AfrRS1) predicts the overlapping chromatograph pattern of the most frequently observed TpiH pattern. Based on the assumption that TpiH specimens carry one TpiR copy we estimate that TpiR represents about $7 \%$ of the Tpi chromosomes in Myanmar, a frequency similar to that calculated for fall armyworm in India and Africa but 2-3 times lower than that typically observed in Western Hemisphere collections from C-strain hosts (Fig. 5).

Comparisons between the African and Myanmar fall armyworms. Adjacent to the strain diagnostic polymorphisms in the fourth exon of the putative Tpi coding region is a variable length intron that exhibits high sequence variability in Western Hemisphere populations ${ }^{31}$. The 172-bp segment of this intron identified 138 variants from Western Hemisphere collections compared to only six haplotypes from 863 Africa specimens ${ }^{30}$. From the 207 Myanmar specimens examined for the Tpi intron four different haplotypes were identified, each of which is identical to sequences found in Africa (Fig. 6). The frequencies of the haplotypes in the Myanmar collections were similar to that observed in Africa.

Characterization of fall armyworm from southern China. In a preliminary sampling of southern China, a total of 44 fall armyworm specimens were collected from corn plants in four provinces. In this study the COI strain identity was determined using the COIA segment (Fig. 1a) ${ }^{31}$. All specimens were of a single haplotype indicative of COI-RS (Fig. 3). Analysis of the segment of the strain diagnostic Tpi exon also showed a single sequence corresponding to TpiC (Fig. 4).

\section{Discussion}

The genetic evidence from studies of fall armyworm from multiple locations in Africa, India, and now Myanmar and China indicate that these populations share a common and recent origin that derived from a small number of introductions (as few as one) from the Western Hemisphere. The strongest evidence comes from the examination of a highly variable segment of a Tpi intron sequence for which over 200 variants have been identified in the Western Hemisphere. In contrast only four different sequences were found in Myanmar out of 150 specimens 


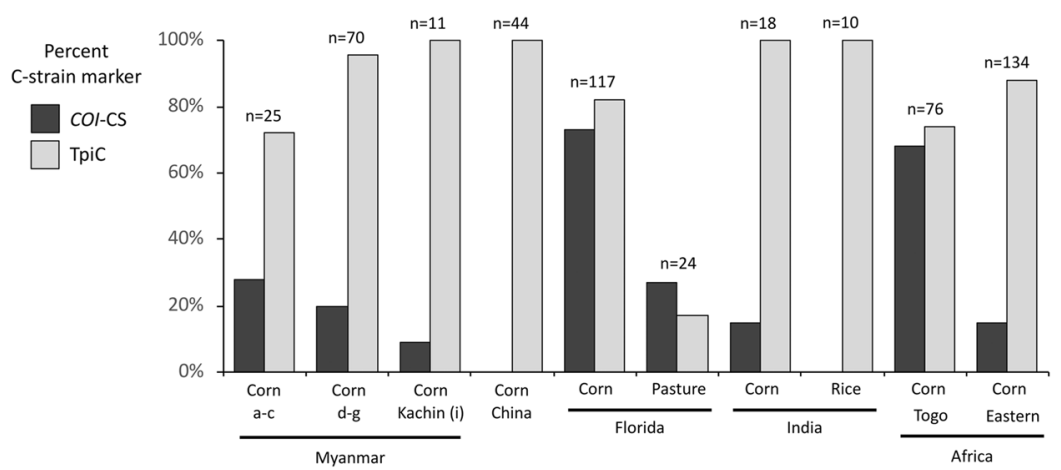

Figure 4. Comparisons of C-strain marker frequencies of larval collections from different locations and host plants. Myanmar sites were grouped by region with letter designations defined in Fig. 1. Eastern Africa is represented by pooled data from Burundi, Kenya, and Tanzania.

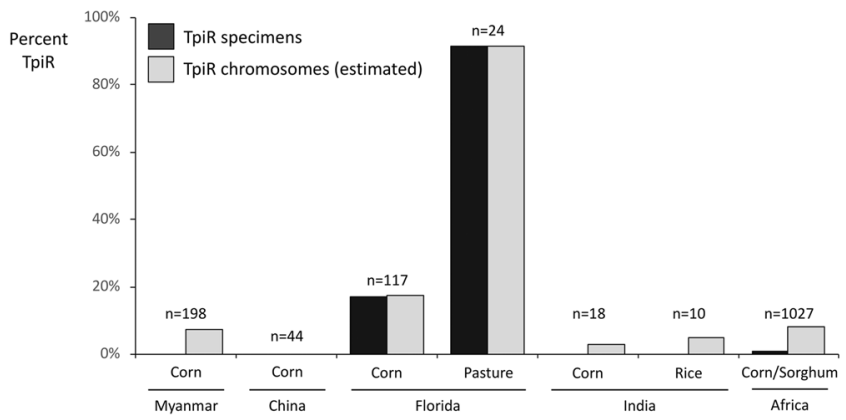

Figure 5. Frequencies of the TpiR haplotype from different regions and host plants calculated on the basis of specimens expressing the haplotype or estimation of chromosome numbers that include contributions from the presumed TpiH interstrain hybrid.

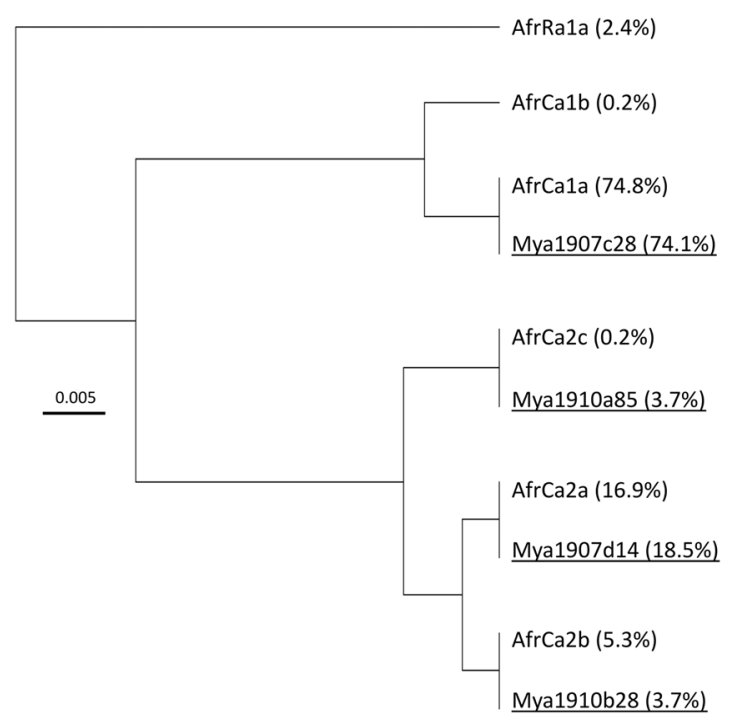

Figure 6. Phylogenetic tree calculated for the TpI4 intron segment using the UPGMA method and Tamura-Nei modeling. Relative frequencies of the different haplotypes are in parentheses.

tested and these are identical to those found in Africa and India (Fig. 6). Additional supporting evidence comes from the similarities in the types and relative frequencies of the COI haplotypes in populations from Myanmar and in particular eastern Africa compared to that typically observed in the Western Hemisphere (Fig. 3b). This includes the predominance of the COI-RS marker in collections from C-strain host plants. Finally, the TpiR haplotype found in the TpiH specimens from Myanmar is identical to the single TpiR variant found in Africa, which has so far not been observed in the Western Hemisphere. This combination of genetic similarity between 
the Eastern Hemisphere fall armyworm populations and low genetic variability is most consistent with a single introduction and subsequent dispersion between Africa and Asia that occurred too recently for the accumulation of sequence polymorphisms. If this scenario is correct it would indicate that the variety of seas, deserts, and mountain ranges that separate Africa and Asia are readily traversed by fall armyworm, suggesting that the hemisphere is at high risk for invasions by migratory moth pests. Estimating the relative contributions of natural and human-assisted (trade and travel) mechanisms for the dispersal of fall armyworm in the Eastern Hemisphere will be essential to assessing the risks of future events of this type and developing effective control strategies.

CLIMEX analysis was used to provide a preliminary estimate of where persistent or permanent fall armyworm populations are most likely to be situated. These could potentially serve as sources for annual migrations in Asia analogous to how North American infestations originate from overwintering locations in south Texas and Florida ${ }^{15,37}$. CLIMEX parameters that produce projections accurately approximating North American overwintering locations show substantial regions in southeastern Asia suitable for fall armyworm populations (Fig. 1a). One collection site in the Hunan province of China (L, Fig. 1a) lies approximately $500 \mathrm{~km}$ from suitable habitats as modeled by CLIMEX, suggesting that the fall armyworm found there were likely to be migrants.

Critical to projections of the range of crops at risk is determining to what extent the R-strain is present in Asia as this subpopulation would be the primary fall armyworm threat to such crops as rice and millet. The available genetic markers are contradictory as the COI-RS haplotype diagnostic of the R-strain and the TpiC marker indicative of the C-strain predominate in the collections from Africa, India, and from this study, Asia ${ }^{5,6,16,30}$. There are two lines of evidence that suggest that a single strain predominates in the Eastern Hemisphere and that it is most likely the C-strain. The first is that major infestations have been primarily, if not exclusively, reported in the C-strain preferred host plants corn and sorghum, with genetic characterizations to date limited to collections from these sites. Therefore, the TpiC marker is displaying the expected correspondence with host plant preference. Second, a methodology was developed that could detect suppression of interstrain mating in Western Hemisphere field populations by comparing the frequency of heterozygosity between strain-specific genetic polymorphisms with those that are nonspecific ${ }^{38}$. Application of this method to African fall armyworm found no evidence of similar strain-dependent mating behavior ${ }^{39}$. Third, previous studies in the Western Hemisphere indicate that approximately $20 \%$ of fall armyworm larvae collected from corn are of the R-strain based on a variety of molecular markers ${ }^{17,21,25,40}$, including TpiR (Fig. 5). In contrast, only 11 TpiR specimens have been detected in the total of 1297 samples so far analyzed from the Eastern Hemisphere. At this time, the only evidence for TpiR in India, Myanmar, and China is from TpiH heterozygotes. Overall, these observations suggest that the African fall armyworm is behaving as expected for the C-strain, with the R-strain a minor presence or perhaps even absent.

In Myanmar, China, India, and most of Africa, the COI strain marker is in disagreement with both Tpi and host plant. One way this could have occurred is if the linkage between the mitochondrial COI marker and strain identity was disrupted by interstrain mating. For example, since mitochondria is maternally inherited, mating between an R-strain female and C-strain male would produce COI-RS hybrid daughters, which if they also mated with C-strain males would produce COI-RS progeny in a C-strain (including TpiC) background. Under this scenario the originating population entering the Eastern Hemisphere carried both strains with interstrain hybridization occurring as described above. If chance or circumstance caused the R-strain subgroup to diminish then the predominating $\mathrm{C}$-strain would still be associated with TpiC but would now a mixture of the COI markers. If correct then the COI marker is no longer strain-specific in the Eastern Hemisphere populations, leaving only the $\mathrm{TpiC}$ haplotype as a diagnostic molecular marker of strain identity.

Fall armyworm in the Eastern Hemisphere appears to be recently arrived and in the process of rapid dispersion. If so, we can expect substantial changes in haplotype frequency and distribution as populations equilibrate and more extensive and systematic monitoring are performed. The current study provides a snapshot of the surveyed populations in Myanmar and parts of China in 2018-2019, providing a genetic baseline for future comparisons. Of particular interest and economic relevance is whether the R-strain, the fall armyworm subpopulation believed to be the biggest threat to rice and millet, is present in Asia. Even if it is not, the apparent rapidity and extent of the current fall armyworm invasion is a warning of how quickly the R-strain could become widely disseminated in Asia if introduced. Similarly, fall armyworm in the Western Hemisphere can exhibit resistance to several insecticidal proteins from the bacterium Bacillus thuringiensis $(\mathrm{Bt})$ that are used in transgenic corn lines $^{41-43}$. At this time, there is no evidence that these resistance traits are present in Africa ${ }^{6}$. These observations underscore the importance of understanding the migratory history of fall armyworm in the Eastern Hemisphere in order to prevent or slow future introductions of the R-strain (if not already present) or other fall armyworm subpopulations from the Western Hemisphere known to carry pesticide resistance or other deleterious traits.

\section{Data availability}

All data generated or analyzed during this study are included in this published article.

Received: 25 October 2019; Accepted: 13 January 2020;

Published online: 29 January 2020

\section{References}

1. Luginbill, P. The fall armyworm. U.S. Dept. Agric. Tech. Bull. 34, 1-91 (1928).

2. Goergen, G., Kumar, P. L., Sankung, S. B., Togola, A. \& Tamo, M. First report of outbreaks of the fall armyworm Spodoptera frugiperda (J E Smith) (Lepidoptera, Noctuidae), a new alien invasive pest in west and central Africa. PLoS One 11 (2016).

3. Cock, M.J.W., Beseh, P.K., Buddie, A.G., Cafa, G. \& Crozier, J. Molecular methods to detect Spodoptera frugiperda in Ghana, and implications for monitoring the spread of invasive species in developing countries. Scientific Reports 7 (2017).

4. Jacobs, A., van Vuuren, A. \& Rong, I. H. Characterisation of the fall armyworm (Spodoptera frugiperda JE Smith) (Lepidoptera: Noctuidae) from South Africa. African Entomology 26, 45-49 (2018).

5. Nagoshi, R. N. et al. Analysis of strain distribution, migratory potential, and invasion history of fall armyworm populations in northern Sub-Saharan Africa. Scientific Reports 8, 3710 (2018). 
6. Nagoshi, R. N. et al. Comparative molecular analyses of invasive fall armyworm in Togo reveal strong similarities to populations from the eastern United States and the Greater Antilles. Plos One 12, e0181982 (2017).

7. Srinivasan, R., Malini, P. \& Othim, S. T. O. Fall armyworm in Africa: which 'race' is in the race, and why does it matter? Current Science 114, 27-28 (2018).

8. Ganiger, P. C. et al. Occurrence of the new invasive pest, fall armyworm, Spodoptera frugiperda (JE Smith) (Lepidoptera: Noctuidae), in the maize fields of Karnataka, India. Current Science 115, 621-623 (2018).

9. Sharanabasappa et al. First report of fall armyworm, Spodoptera frugiperda (JE Smith) (Lepidoptera: Noctuidae), a new invasive alien pest of maize in India. Pest Management in Horticultural Ecosystems 24, 23-29 (2018).

10. Shylesha, A. N. et al. Studies on new invasive pest Spodoptera frugiperda (J. E. Smith) (Lepidoptera: Noctuidae) and its natural enemies. Journal of Biological Control 32, 145-151 (2018).

11. Swamy, H. M. M. et al. Prevalence of "R" strain and molecular diversity of fall armyworm Spodoptera frugiperda (J.E. Smith) (Lepidoptera: Noctuidae) in India. Indian Journal of Entomology 80, 544-553 (2018).

12. Jing, D. P. et al. Initial detections and spread of invasive Spodoptera frugiperda in China and comparisons with other noctuid larvae in cornfields using molecular techniques. Insect Sci (2019).

13. Li, X. J. et al. Prediction of migratory routes of the invasive fall armyworm in eastern China using a trajectory analytical approach. Pest Manag Sci (2019).

14. Yee, K. H. et al. First Detection Report of the Fall Armyworm Spodoptera frugiperda (Lepidoptra: Noctuidae) on Maize in Myanmar. IPPC Official Pest Report No. MMR-19/2, https://www.ippc.int/static/media/files/pestreport/2019/01/11/Detection__report_of_ FAW_in_Myanmar.pdf (2019).

15. Westbrook, J. K., Nagoshi, R. N., Meagher, R. L., Fleischer, S. J. \& Jairam, S. Modeling seasonal migration of fall armyworm moths. International Journal of Biometeorology 60, 255-267 (2016).

16. Nagoshi, R. N. et al. Genetic characterization of fall armyworm infesting South Africa and India indicate recent introduction from a common source population. PLoS One 14, e0217755 (2019).

17. Murúa, M. G. et al. Demonstration using field collections that Argentina fall armyworm populations exhibit strain-specific host plant preferences. Journal of Economic Entomology 108, 2305-2315 (2015).

18. Nagoshi, R. N. \& Meagher, J. R. L. Behavior and distribution of the two fall armyworm host strains in Florida. Florida Entomologist 87, 440-449 (2004).

19. Pashley, D. P. Quantitative genetics, development, and physiological adaptation in host strains of fall armyworm. Evolution 42, 93-102 (1988).

20. Juárez, M. L. et al. Host association of Spodoptera frugiperda (Lepidoptera: Noctuidae) corn and rice strains in Argentina, Brazil, and Paraguay. Journal of Economic Entomology 105, 573-582 (2012).

21. Nagoshi, R. N. The fall armyworm triose phosphate isomerase (Tpi) gene as a marker of strain identity and interstrain mating. Annals of the Entomological Society of America 103, 283-292 (2010).

22. Nagoshi, R. N. Improvements in the identification of strains facilitate population studies of fall armyworm subgroups. Annals of the Entomological Society of America 105, 351-358 (2012).

23. Meagher, R. L. \& Nagoshi, R. N. Population dynamics and occurrence of Spodoptera frugiperda host strains in southern Florida. Ecological Entomology 29, 614-620 (2004).

24. Nagoshi, R. N. \& Meagher, R. L. Seasonal distribution of fall armyworm (Lepidoptera: Noctuidae) host strains in agricultural and turf grass habitats. Environmental Entomology 33, 881-889 (2004).

25. Prowell, D. P., McMichael, M. \& Silvain, J. F. Multilocus genetic analysis of host use, introgression, and speciation in host strains of fall armyworm (Lepidoptera: Noctuidae). Annals of the Entomological Society of America 97, 1034-1044 (2004).

26. PPD. Insect pests of corn in Myanmar. Plant Protection Division Myanmar Agriculture Service, https://ppdmyanmar.org/insectpests-of-corn/ (2020).

27. Tamura, K. \& Nei, M. Estimation of the number of nucleotide substitutions in the control region of mitochondrial-DNA in humans and chimpanzees. Molecular Biology and Evolution 10, 512-526 (1993).

28. Kearse, M. et al. Geneious Basic: An integrated and extendable desktop software platform for the organization and analysis of sequence data. Bioinformatics 28, 1647-1649 (2012).

29. Nagoshi, R. N. et al. Fall armyworm migration across the Lesser Antilles and the potential for genetic exchanges between North and South American populations. Plos One 12, e0171743, https://doi.org/10.1371/journal.pone.0171743 (2017).

30. Nagoshi, R. N., Goergen, G., Plessis, H. D., van den Berg, J. \& Meagher, R. Jr. Genetic comparisons of fall armyworm populations from 11 countries spanning sub-Saharan Africa provide insights into strain composition and migratory behaviors. Sci Rep $\mathbf{9}, 8311$ (2019).

31. Nagoshi, R. N. \& Meagher, R. L. Using intron sequence comparisons in the triose-phosphate isomerase gene to study the divergence of the fall armyworm host strains. Insect Molecular Biology 25, 324-337 (2016).

32. Kriticos, D. J. et al. CLIMEX Version 4: Exploring the effects of climate on plants, animals and diseases., (CSIRO, Canberra, 2015).

33. Ramirez-Cabral, N. Y. Z., Kumar, L. \& Shabani, F. Future climate scenarios project a decrease in the risk of fall armyworm outbreaks. Journal of Agricultural Science 155, 1219-1238 (2017).

34. Kriticos, D. J. et al. CliMond: global high-resolution historical and future scenario climate surfaces for bioclimatic modelling. Methods in Ecology and Evolution 3, 53-64 (2012).

35. Pair, S. D. \& Sparks, A. N. Evidence of annual long distance migration by the fall armyworm. in Long-range migration of moths of agronomic importance to the United States and Canada: Specific examples of occurrence and synoptic weather patterns conducive to migration (ESA Symposium, 1982). Vol. ARS-43 (ed. Sparks, A. N.) 25-33 (U.S. Department of Agriculture, Misc. Publ., Washington, D.C., 1986)

36. Nagoshi, R. N. et al. Haplotype Profile Comparisons Between Spodoptera frugiperda (Lepidoptera: Noctuidae) Populations From Mexico With Those From Puerto Rico, South America, and the United States and Their Implications to Migratory Behavior. Journal of Economic Entomology 108, 135-144 (2015).

37. Nagoshi, R. N., Fleischer, S. J. \& Meagher, R. L. Texas is the overwintering source of fall armyworm in central Pennsylvania: Implications for migration into the northeastern United States. Environmental Entomology 38, 1546-1554 (2009).

38. Nagoshi, R. N., Fleischer, S. \& Meagher, R. L. Demonstration and quantification of restricted mating between fall armyworm host strains in field collections by SNP comparisons. Journal of Economic Entomology 110, 2568-2575 (2017).

39. Nagoshi, R. N. Evidence that a major subpopulation of fall armyworm found in the Western Hemisphere is rare or absent in Africa, which may limit the range of crops at risk of infestation. PLoS One 14, e0208966 (2019).

40. Nagoshi, R. N., Silvie, P., Meagher, R. L., Lopez, J. \& Machados, V. Identification and comparison of fall armyworm (Lepidoptera: Noctuidae) host strains in Brazil, Texas, and Florida. Annals of the Entomological Society of America 100, 394-402 (2007).

41. Adamczyk, J. J. \& Sumerford, D. V. Increased tolerance of fall armyworms (Lepidoptera: Noctuidae) to Cryl Ac delta-endotoxin when fed transgenic Bacillus thuringiensis cotton: Impact on the development of subsequent generations. Florida Entomologist 84, $1-6(2001)$.

42. Banerjee, R. et al. Mechanism and DNA-based detection of field-evolved resistance to transgenic Bt corn in fall armyworm (Spodoptera frugiperda). Sci Rep 7, 10877 (2017).

43. Storer, N. P. et al. Discovery and characterization of field resistance to Bt maize: Spodoptera frugiperda (Lepidoptera:Noctuidae) in Puerto Rico. Journal of Economic Entomology 103, 1031-1038 (2010). 


\section{Acknowledgements}

We recognize Dr. J.M.G. Thomas for technical assistance in preparing the specimens. We thank Dr. Anna Block and Dr. Seth Britch for comments about the manuscript. Support came from the Agricultural Research Service and the Agency for International Development of the United States Department of Agriculture. We appreciate the logistics support of Dr. Ye Tint Tun Director General of the Department of Agriculture, and Aung Kyaw Oo Director of the Plant Protection Division (PPD), Myanmar. We thank the staff of the PPD Myanmar for performing the larvae collection. Dr. Karim Maredia from Michigan State University was a key person to establish FAW collaboration at Myanmar. We are grateful to National Agro-Tech Extension and Service Center in China for field assistance. The use of trade, firm, or corporation names in this publication is for the information and convenience of the reader. Such use does not constitute an official endorsement or approval by the United States Department of Agriculture or the Agricultural Research Service of any product or service to the exclusion of others that may be suitable.

\section{Author contributions}

R.N.N., D.B., D.M.-S., Y.X. designed the experiment; N.N.H., D.M.-S., L.Z., Y.X. collected field specimens and field data; R.N.N., L.Z., Y.X. produced the genetic data and analyzed the results; B.Y.N. produced CLIMEX modeling analysis. R.N.N., L.Z. wrote the initial draft of the manuscript; all authors reviewed and edited the manuscript.

\section{Competing interests}

The authors declare no competing interests.

\section{Additional information}

Correspondence and requests for materials should be addressed to R.N.N.

Reprints and permissions information is available at www.nature.com/reprints.

Publisher's note Springer Nature remains neutral with regard to jurisdictional claims in published maps and institutional affiliations.

(c) (i) Open Access This article is licensed under a Creative Commons Attribution 4.0 International License, which permits use, sharing, adaptation, distribution and reproduction in any medium or format, as long as you give appropriate credit to the original author(s) and the source, provide a link to the Creative Commons license, and indicate if changes were made. The images or other third party material in this article are included in the article's Creative Commons license, unless indicated otherwise in a credit line to the material. If material is not included in the article's Creative Commons license and your intended use is not permitted by statutory regulation or exceeds the permitted use, you will need to obtain permission directly from the copyright holder. To view a copy of this license, visit http://creativecommons.org/licenses/by/4.0/.

(C) The Author(s) 2020 